\title{
Oxaloacetate decarboxylase of Archaeoglobus fulgidus Cloning of genes and expression in Escherichia coli
}

\author{
Journal Article \\ Author(s): \\ Dahinden, Pius; Pos, Klaas M.; Taralczak, Malgorzata; Dimroth, Peter \\ Publication date: \\ 2004-11 \\ Permanent link: \\ https://doi.org/10.3929/ethz-b-000160275 \\ Rights / license: \\ In Copyright - Non-Commercial Use Permitted \\ Originally published in: \\ Archives of Microbiology 182(5), https://doi.org/10.1007/s00203-004-0706-0
}




\section{Pius Dahinden · Klaas M. Pos $\cdot$ Malgorzata Taralczak Peter Dimroth \\ Oxaloacetate decarboxylase of Archaeoglobus fulgidus: cloning of genes and expression in Escherichia coli}

\begin{abstract}
Archaeoglobus fulgidus harbors three consecutive and one distantly located gene with similarity to the oxaloacetate decarboxylase $\mathrm{Na}^{+}$pump of Klebsiella pneumoniae (KpOadGAB). The water-soluble carboxyltransferase (AfOadA) and the biotin protein (AfOadC) were readily synthesized in Escherichia coli, but the membrane-bound subunits AfOadB and AfOadG were not. AfOadA was affinity purified from inclusion bodies after refolding and AfOadC was affinity purified from the cytosol. Isolated AfOadA catalyzed the carboxyltransfer from $\left[4-{ }^{14} \mathrm{C}\right]$-oxaloacetate to the prosthetic biotin group of AfOadC or the corresponding biotin domain of KpOadA. Conversely, the carboxyltransferase domain of KpOadA exhibited catalytic activity not only with its pertinent biotin domain but also with AfOadC.
\end{abstract}

Keywords Archaeoglobus fulgidus - Oxaloacetate decarboxylase $\cdot \mathrm{Na}^{+}$pump $\cdot$ Archaeal membrane protein $\cdot$ Protein targeting and translocation .

Refolding of inclusion bodies

\begin{abstract}
Abbreviations Af: Archaeoglobus fulgidus - AHT: Anhydrotetracycline - DTT: Dithiothreitol - IPTG: Isopropyl- $\beta$-D-thiogalactopyranoside $\cdot \mathrm{Kp}$ : Klebsiella pneumoniae - LDAO: $\quad N, N$-dimethyldodecylamineN-oxide MBP: Maltose binding protein . OAD: Oxaloacetate decarboxylase - OadA: Oxaloacetate decarboxylase $\alpha$-subunit OadB: Oxaloacetate decarboxylase $\beta$-subunit - OadC: Oxaloacetate decarboxylase $\delta$-subunit - OadG: Oxaloacetate decarboxylase $\gamma$-subunit $\cdot$ PPS: 3-(1-pyridino)-1-propanesulfate
\end{abstract}

P. Dahinden · K. M. Pos · M. Taralczak · P. Dimroth $(\bowtie)$ Institut für Mikrobiologie der ETH Zürich,

ETH-Zentrum, Schmelzbergstrasse 7, 8092 Zürich, Switzerland

E-mail: dimroth@micro.biol.ethz.ch

Tel.: +41-1-6323321

Fax: + 41-1-6321378

\section{Introduction}

Genome sequencing of Archaeoglobus fulgidus (Af) (Klenk et al. 1997) revealed a cluster of three open reading frames (ORFs) with high similarity to genes encoding subunits of the $\mathrm{Na}^{+}$translocating decarboxylase enzyme family (for reviews see Dimroth 1997; Buckel 2001). Accordingly, the gene products of the ORFs AF2084, AF2085 and AF2086 were identified as the membrane-integral carboxybiotin decarboxylase ( $\beta$-subunit), the biotin-binding protein and the membrane-anchored $\gamma$-subunit, respectively. At separate locations, genes encoding carboxyltransferases with specificities for oxaloacetate (AF1252) or methylmalonyl-CoA (AF2216) are found. The latter gene is succeeded by AF2217, encoding a biotin carrier protein. Based on this information, one may conclude that A. fulgidus has the capacity to synthesize an oxaloacetate decarboxylase and a methylmalonyl-CoA decarboxylase $\mathrm{Na}^{+}$pump and that these enzyme complexes share part of their subunits with each other.

Most of the fundamental biochemistry of the $\mathrm{Na}^{+}$ translocating decarboxylases has been studied with the oxaloacetate decarboxylase from Klebsiella pneumoniae (KpOAD). The catalytic cycle starts with the carboxyltransfer from oxaloacetate to the biotin group on KpOadA-C. Subsequently, KpOadB catalyzes the decarboxylation of carboxybiotin coupled to the transport of two $\mathrm{Na}^{+}$ions into the periplasmic reservoir and consumption of a periplasmically derived proton ( $\mathrm{Di}$ Berardino and Dimroth 1996). By mutational studies, three amino acids (D203, Y229, S382) were identified to have a key function in the ion-transport-coupled decarboxylation event (Jockel et al. 2000a,b; Schmid et al. 2002a). The most highly conserved portion of the protein is helix VIII, which provides part of the ion channel (Wild et al. 2003). KpOadG harbors a $\mathrm{Zn}^{2+}$ ion that is part of the carboxyltransferase catalytic site and it is essential for the formation of a stable enzyme complex (Schmid et al. 2002b). 
For detailed insights into the catalytic mechanism of the $\mathrm{Na}^{+}$pump, structural information is of essence. Progress in this direction was obtained recently with the crystal structure of the carboxyltransferase subunit of glutaconyl-CoA decarboxylase (Wendt et al. 2003). With the same aim we have investigated oxaloacetate decarboxylase from the thermophile A. fulgidus. Here, we report on the expression of the genes AfoadA and $A f$ oadC in Escherichia coli and on the purification and characterization of the gene products.

\section{Materials and methods}

Strains and growth conditions Archaeoglobus fulgidus VC-16 (DSM 4304) was grown anaerobically at $83^{\circ} \mathrm{C}$ in sulfate-thiosulfate-lactate (STL) medium [medium 3, (Balch et al. 1979)] gassed with $\mathrm{N}_{2} / \mathrm{CO}_{2} \quad(80 \%: 20 \%$, $200 \mathrm{kPa})$.

Recombinant DNA techniques and sequencing Standard microbiological methods were used as described (Ausubel et al. 1989; Sambrook et al. 1989). Polymerase chain reaction (PCR) was done with $P f u$ polymerase and $A$. fulgidus chromosomal DNA as template. All inserts derived from PCR as well as the ligation sites were checked by DNA sequencing (Sanger et al. 1977).

Cloning of the Afoad genes The oligonucleotide primers 5'-TCTGCATCTGGCTCATATGGCAAAAG-3' (AfOA-NdeI) and 5'-ATATTTAGTCGCTCGAGCTT TTCTGGC-3' (AfOA-XhoI-Hi), 5'-CGATCTAGCTCA TATGCTCGGAAGCCTCG-3' (AfOB- $N d e \mathrm{I}$ ) and 5'-CCTATTCGATCTCGAGCCCAAGAATCTG-3' (AfOB-XhoI-Hi), 5'-CGATCTAGCTCATATGAGGT TTTACAG-3' (AfOC-NdeI) and 5'-GCTATTCGATC TCGAGGCTCACCGGCTGG-3' (AfOC-XhoI-Hi), all including an $\mathrm{NdeI}$ or $\mathrm{XhoI}$ site (underlined), were used to amplify AF1252, AF2084 and AF2085, respectively. PCR products were digested with $N d e \mathrm{I}$ and $X h o \mathrm{I}$ and ligated to the adequately digested, dephosphorylated vector $\mathrm{pET} 24 \mathrm{~b}$ (Novagen). The resulting plasmids were designated pET24b-AfoadA, pET24b-AfoadB or pET24b-AfoadC, respectively, and all encoded the respective subunit including a C-terminal His-tag.

With the oligonucleotide primers AfOA-NdeI (see above) and 5'-AATGTCTCGCTCGAGCCACCTTGTCG-3' (AfOA-XhoI) AfoadA was amplified, whereas $A$ foadB was amplified with AfOB-NdeI and 5'CCTATTCGATCTCGAGCTACCCAAGAATCTG-3' (AfOB-XhoI). The resulting PCR products included $N d e \mathrm{I}$ and $X h o \mathrm{I}$ restriction sites which were used for the unidirectional cloning into $\mathrm{pET} 16 \mathrm{~b}$ vector to obtain pET16b-AfoadA or pET16b-AfoadB, encoding oxaloacetate decarboxylase subunits $\alpha$ and $\beta$ including an $\mathrm{N}$-terminal His-tag.

With the oligonucleotide primers 5'-TCAATCGGTCTCGGCGCCATGCTCGGAAGCCTCGTG-3'
(AfOB-Str fo) and 5'-ACCCTCGGTCTCTTATCAC TACCCAĀGAATCTGAATC-3' (AfOB-Str_re), both including a $B s a \mathrm{I}$ site (underlined), $A$ foadB was amplified from chromosomal A. fulgidus DNA. The PCR product and the vector pASK-IBA4 (IBA $\mathrm{GmbH}$ ) were digested with $B s a \mathrm{I}$. The digested and ligated fragments resulted in plasmid pASK-AfoadB, encoding an A. fulgidus oxaloacetate decarboxylase $\beta$-subunit including an $\mathrm{N}$-terminal Strep-tag.

To obtain a plasmid in which all putative Afoad genes are under the control of one promoter, the ORFs AF2086 ( $\gamma$-subunit), AF2085 and AF2084 were amplified with the oligonucleotide primers 5'-ATCTCGGTATGACATATGATAGATTTGGCCATAA TGCTCACTGTAGAGG-3' (AfOadGCB- $N d e \mathrm{I}$ ) and 5'-TC ATAGTATGAGCTCTCAGCTAGCCAAAAT GCGATGATTAACCTACCC-3' (AfOadGCB-SacI), including $N d e \mathrm{I}$ and $S a c \mathrm{I}$ sites (underlined). ORF AF1252 was amplified with the oligonucleotide primers 5'-TGTAGTATTGAGCTCTGAGGATCCATTTCCC ATCAGGCTTGAGACATGGC-3' (AfOadA-SacI) and 5'-TGATATATCCTCGAGTATGCGGCCGCAAGAGGTATATCACTTTTCTGGC-3' (AfOadAXhoI), including $S a c \mathrm{I}$ and XhoI sites (underlined). Plasmid pET24b-AfoadGCBA was obtained by ligation of the accordingly digested PCR products and vector pET24b.

Growth of recombinant E. coli and expression of genes For expression of the Afoad genes, baffled Erlenmeyer flasks containing LB $(200 \mathrm{ml}$ to 21$)$ were inoculated 1:100 with an overnight culture and incubated at $37^{\circ} \mathrm{C}$ at $180 \mathrm{rpm}$ until the culture reached an $\mathrm{OD}_{600}$ of approximately 0.6 . After cooling to $4^{\circ} \mathrm{C}$, the expression of the genes was induced by the addition of IPTG $(100 \mu \mathrm{M})$ and the culture was incubated for another $4 \mathrm{~h}$ at $37^{\circ} \mathrm{C}$ and shaking at $180 \mathrm{rpm}$ before harvesting the cells. In the case of $\mathrm{AfOadA}_{\mathrm{His}}$ synthesis, the cultures were grown at $30^{\circ} \mathrm{C}$ before induction and at $25^{\circ} \mathrm{C}$ after induction.

Refolding of AfOadA from inclusion bodies Cells containing inclusion bodies were resuspended in $5 \mathrm{ml}$ per gram of cells $50 \mathrm{mM}$ Tris- $\mathrm{HCl}, \mathrm{pH} 7.0,1 \mathrm{mM}$ EDTA and $3 \mathrm{mM} \mathrm{MgCl}_{2}$. After the addition of $7.5 \mathrm{mg}$ lysozyme and $50 \mu \mathrm{g}$ DNase I, the suspension was incubated for $25 \mathrm{~min}$ at $25^{\circ} \mathrm{C}$ and then passed three times through a French pressure cell at $110 \mathrm{MPa}$. Subsequently, $20 \mathrm{mM}$ EDTA, $\mathrm{pH}$ 8.0, $0.5 \mathrm{M} \mathrm{NaCl}$ and 2\% Triton X-100 (final concentration) were added and the mixture incubated for $30 \mathrm{~min}$ at $4^{\circ} \mathrm{C}$. Inclusion bodies were harvested by centrifugation at $20,000 \times g$ and washed twice with $100 \mathrm{mM}$ Tris- $\mathrm{HCl}, \mathrm{pH} 7.5$ and $20 \mathrm{mM}$ EDTA $(10 \mathrm{ml})$, before they were solubilized with $100 \mathrm{mM}$ Tris- $\mathrm{HCl}, \mathrm{pH}$ 8.0, $1 \mathrm{mM}$ EDTA, $6 \mathrm{M}$ guanidinium chloride and $100 \mathrm{mM}$ dithiothreitol (at $10 \mathrm{mg}$ protein per $\mathrm{ml}$ ). After $2-3 \mathrm{~h}$ at $25^{\circ} \mathrm{C}$, solubilization was complete. After adjusting the $\mathrm{pH}$ to $3-4$ with $10 \mathrm{mM} \mathrm{HCl}$ and centrifugation, the supernatant $(0.5 \mathrm{ml})$ was slowly diluted into 
$50 \mathrm{ml} 100 \mathrm{mM}$ Tris-HCl, $1 \mathrm{mM}$ EDTA, $1 \mathrm{mM}$ DTT containing $500 \mathrm{mM}$ L-arginine or $1 \mathrm{M}$ 3-(1-pyridino)-1propanesulfate (PPS) and/or $0.1 \%$ LDAO (adjusted to a final $\mathrm{pH}$ of 8.5 ) at $15^{\circ} \mathrm{C}$. After $10 \mathrm{~min}$, another $0.5 \mathrm{ml}$ of solubilized protein solution was slowly added. This cycle was repeated 19 times to reach a final guanidinium chloride concentration of just below $1 \mathrm{M}$. Precipitated protein was removed by passage through a $0.22-\mu \mathrm{m}$ filter. Soluble, but higher aggregates of AfOadA were removed by precipitation with $40 \%$ ammonium sulfate. Soluble protein was then precipitated with $60 \%$ ammonium sulfate and the precipitate collected by centrifugation. The protein was dissolved in $20 \mathrm{mM}$ Tris- $\mathrm{HCl}$, pH $8.0,100 \mathrm{mM} \mathrm{NaCl}$ and $0.1 \%$ LDAO to a concentration of about $1 \mathrm{mg} / \mathrm{ml}$, dialyzed overnight against 21 of $20 \mathrm{mM}$ Tris- $\mathrm{HCl}$, pH 8.0, containing $100 \mathrm{mM} \mathrm{NaCl}$ and subsequently passed through a $0.22-\mu \mathrm{m}$ filter unit.

Preparation of cytosolic fraction and membranes Cells from expression cultures $(1 \mathrm{~g}$ wet weight) were suspended in $5 \mathrm{ml}$ of a suitable buffer containing $0.2 \mathrm{mM}$ DFP and $50 \mu \mathrm{g}$ DNase I. The cells were disrupted by three passages through a French pressure cell at $110 \mathrm{MPa}$. Intact cells and cell debris were removed by centrifugation $(30 \mathrm{~min}$ at $8,000 \times g$ ), and the cell-free supernatant was subjected to ultracentrifugation $(1 \mathrm{~h}$ at $200,000 \times g$ ) to separate the cytosolic fraction and the membrane fraction.

Purification of AfOadA $A_{\text {His }}$ by $\mathrm{Ni}^{2+}-\mathrm{NTA}$ chromatography The $\alpha$-subunit was synthesized in E. coli C43(DE3) (Miroux and Walker 1996) harboring pET24b-AfoadA or pET16b-AfoadA. The cells were resuspended in HisBind buffer $(20 \mathrm{mM}$ Tris- $\mathrm{HCl}, \mathrm{pH} 8.0$, and $500 \mathrm{mM}$ $\mathrm{NaCl})$. The cytosolic fraction was prepared as described above and applied to a $\mathrm{Ni}^{2+}$-NTA-agarose column ( $2 \mathrm{ml}$ bed volume, Qiagen), pre-equilibrated with 10 bed volumes HisBind buffer containing $20 \mathrm{mM}$ imidazole. The column was washed three times with 10 bed volumes of the same buffer containing 60,70 and $80 \mathrm{mM}$ imidazole, respectively. AfOadA $\mathrm{A}_{\mathrm{His}}$ was then eluted in 2 bed volumes of HisBind buffer containing $250 \mathrm{mM}$ imidazole.

Purification of AfOadC by affinity chromatography using monomeric avidin-Sepharose The $\delta$-subunit was synthesized in E. coli C43(DE3) harboring pET24b-AfoadC in the presence of $10 \mathrm{mM}(+)$-D-biotin. The cells were resuspended in buffer A $(20 \mathrm{mM}$ Tris- $\mathrm{HCl}, \mathrm{pH} 8.0$, $500 \mathrm{mM} \mathrm{NaCl}$, and $1 \mathrm{mM} \mathrm{MgK}_{2}$ EDTA). The cytosolic fraction was prepared as described above and applied to a SoftLink Soft Release avidin resin column (Promega) (Dimroth 1986). The column was washed with 7 bed volumes of buffer B (20 mM Tris- $\mathrm{HCl}, \mathrm{pH} 8.0,500 \mathrm{mM}$ $\mathrm{NaCl}$ ), and $\mathrm{AfOadC}$ was eluted with 2 bed volumes of buffer B containing $5 \mathrm{mM}(+)$-D-biotin.

Carboxyltransfer from oxaloacetate to the biotin-containing subunit The carboxyltransferase activity was measured with $\left[4-{ }^{14} \mathrm{C}\right]$-oxaloacetate and AfOadA and AfOadC or KpOadA-C, essentially as described before (Di Berardino and Dimroth 1995; Jockel et al. 2000).

\section{Results and discussion}

Identification, cloning and expression of genes similar to oadGAB of $K$. pneumoniae

Oxaloacetate decarboxylase of $K$. pneumoniae (KpOadGAB) is the prototype for the $\mathrm{Na}^{+}$transporting carboxylic acid decarboxylase (NaT-DC) family (Busch and Saier 2003). All members of this family contain a membrane-bound $\beta$-subunit involved in $\mathrm{Na}^{+}$translocation and a peripheral $\alpha$-subunit. In oxaloacetate decarboxylase of $K$. pneumoniae, the $\alpha$-subunit consists of the carboxyltransferase domain (OadA-N) and the biotin-containing $\mathrm{CO}_{2}$ acceptor domain (OadA-C). Most members of the NaT-DC family have an additional small membrane-anchored subunit (designated $\gamma$ or $\delta$ ) which is required for the formation of a stable enzyme complex (Huder and Dimroth 1993; Bott et al. 1997; Schmid et al. 2002). A database search for proteins related to $\mathrm{KpOadB}$ resulted in the identification of 27 similar sequences from eubacteria and four from archaea. Because of our interest in the structure of the decarboxylase and the consideration that proteins from thermophilic sources might be more stable under ambient crystallization conditions, we decided to select and amplify the genes encoding the subunits of the putative oxaloacetate decarboxylase from A. fulgidus (Table 1). Based on homology searches with KpOAD, A. fulgidus encodes a putative oxaloacetate decarboxylase consisting of subunits $\alpha, \beta, \delta$ and $\gamma$ (ORFs AF1252, AF2084, AF2085, AF2086). See Table 1 and Fig. 1 for details. In $K$. pneumoniae, the oadGAB genes are clustered in an operon that is part of the citrate fermentation regulon (Bott et al. 1995). In contrast, A. fulgidus harbors the Afoad $G C B$ genes and the AfoadA gene at separate locations on its genome (Fig. 1). In addition to the genes for a putative oxaloacetate decarboxylase, two ORFs were found with similarity to the methylmalonyl-CoA decarboxylase subunits $\alpha$ (PmMmdA, carboxyltransferase) and $\gamma$ (PmMmdC, biotin-binding protein) of Propionigenium modestum. These genes were again not clustered with the genes for the membrane-bound subunits. The subunits encoded by the separate $A$ foad $G C B$ gene cluster may therefore be used for both enzymes, the oxaloacetate and the methylmalonyl-CoA decarboxylase $\mathrm{Na}^{+}$pumps.

Oxaloacetate decarboxylase is specifically required for the citrate or tartrate fermentation pathways. Consequently, the oadGAB genes are clustered either with the genes for citrate lyase and for a citrate transporter (K. pneumoniae or S. typhimurium) or with the genes for tartrate dehydratase (S. typhimurium) (Bott 1997). In A. fulgidus, however, the oadGCB and oadA genes are 
Table 1 Comparison of oxaloacetate decarboxylase subunits of Archaeoglobus fulgidus and Klebsiella pneumoniae. The molecular mass is the theoretical molecular mass, deduced from amino acid sequence. The Blosum62 similarity matrix was used to compute identities/similarities by BioEdit (Hall 1999). Values are given in \% identity/similarity

\begin{tabular}{|c|c|c|c|c|c|}
\hline \multirow[t]{2}{*}{ Subunit } & \multicolumn{3}{|l|}{ A. fulgidus } & \multicolumn{2}{|l|}{ K. pneumoniae } \\
\hline & $\begin{array}{l}\text { Molecular mass } \\
(\mathrm{kDa})\end{array}$ & $\begin{array}{l}\text { Amino } \\
\text { acids }\end{array}$ & $\begin{array}{l}\text { Similarity to KpOAD } \\
\text { (identities/similarities) }\end{array}$ & $\begin{array}{l}\text { Molecular mass } \\
(\mathrm{kDa})\end{array}$ & $\begin{array}{l}\text { Amino } \\
\text { acids }\end{array}$ \\
\hline $\operatorname{OadA}(\alpha)$ & 53.9 & 480 & $50 / 67^{\mathrm{a}}$ & 63.4 & 596 \\
\hline $\operatorname{OadB}(\beta)$ & 36.9 & 355 & $48 / 62$ & 44.9 & 433 \\
\hline OadC $(\delta)$ & 15.8 & 143 & $24 / 34^{\mathrm{b}}$ & - & - \\
\hline $\operatorname{OadG}(\gamma)$ & 7.6 & 69 & $19 / 32$ & 8.9 & 83 \\
\hline $\operatorname{MmdA}(\alpha)$ & 56.8 & 516 & $16 / 32$ & - & - \\
\hline $\operatorname{MmdC}(\delta)$ & 15.7 & 140 & $28 / 37$ & - & - \\
\hline
\end{tabular}

${ }^{\mathrm{a} A f O a d A}$ or AfMmdA was compared with the transferase domain of KpOadA (N-terminal 497 amino acids)

${ }^{\mathrm{b}} \mathrm{AfOadC}$ or AfMmdC was compared with the biotin carrier domain of KpOadA (C-terminal 99 amino acids)

neither clustered with the genes for citrate lyase or tartrate dehydratase nor could these genes be identified elsewhere on the genome. We conclude therefore that A. fulgidus is unable to perform citrate or tartrate fermentation and, hence, no specific role could be assigned to the oxaloacetate-decarboxylase-encoding genes in a catabolic pathway. This conclusion is consistent with the inability of $A$. fulgidus to grow on citrate as sole carbon and energy source. A. fulgidus cells grown on lactate did not contain any significant amount of oxaloacetate decarboxylase activity; consequently, no decarboxylase complex could be isolated by the standard procedure with avidin-Sepharose affinity chromatography.

As oxaloacetate decarboxylase could not be obtained from wild-type $A$. fulgidus cells, we tried to synthesize the enzyme in E. coli. For this purpose, the appropriate A. fulgidus genes were cloned into $\mathrm{pET}$ vectors and transferred into E. coli BL21(DE3). Synthesis of the desired proteins was analyzed by SDS-PAGE and Western blotting following purification by affinity chromatography via specific tags. The results of Table 2 and Fig. 2 show that pure AfOadA and AfOadC were isolated from recombinant $E$. coli BL21(DE3) harboring pET24-AfoadA and pET24-AfoadC, respectively. Expression of AfoadA resulted mainly in the formation

Fig. 1 ORFs of Archaeoglobus fulgidus encoding proteins with similarity to OAD subunits/domains from Klebsiella pneumoniae. The ORFs AF2084, AF2085, and AF2086 encode proteins similar to $\mathrm{KpOadB}$, KpOadA-C, and $\mathrm{KpOadG}$, respectively, of the OAD from K. pneumoniae (KpOadGAB). The ORF AF1252, located on a remote part of the A. fulgidus genome, is similar to KpOadA-N. The latter is the carboxyltransferase domain of $\mathrm{KpOadA}$, comprising the N-terminal 497 amino acids, whereas KpOadA-C is the biotin-binding domain of KpOadA, comprising the C-terminal 99 amino acids of inclusion bodies, even when the cells were grown at $25^{\circ} \mathrm{C}$. From $1 \mathrm{~g}$ of cells (wet weight), $0.3 \mathrm{mg}$ of AfOadA was affinity purified from the cytoplasm while $100 \mathrm{mg}$ were present in inclusion bodies.

Biotinylation of AfOadC was shown by Western blot analysis and staining with avidin-conjugated alkaline phosphatase. The amount of AfOadC isolated by avidin-Sepharose chromatography was approximately half of that obtained by $\mathrm{Ni}^{2+}$-NTA chromatography; therefore, approximately $50 \%$ of AfOadC was equipped with the biotin prosthetic group. Extensive attempts were made to express AfOadB either individually or in various combinations with AfOadA, AfOadC, and AfOadG using various expression hosts at different growth stages. Details of these experiments, compiled in $\mathrm{Ta}-$ ble 2 , indicated no increase in the expression level under all examined conditions.

We therefore considered the possibility that the presence of rare codons prevented the synthesis of AfOadB in E. coli. However, the mean difference in codon usage for $A$ foadB $(21.5 \%)$, calculated using the E. coli codon usage table with the gcua program (Fuhrmann M, Ferbitz L, Hausherr A, Schrodl T, and Hegemann P, in preparation), did not significantly deviate from the value for AfoadA (20.5\%), which was synthesized in large amounts (100 mg/g cells). Moreover, no expression of $A$ foadB was detected with expression hosts containing extra copies of tRNAs recognizing rare codons.

Since specifically the two membrane-bound proteins AfOadB and AfOadG were not successfully synthesized in E. coli, their insertion and/or proper folding into the native structure could have been the main problem. For most membrane proteins of $E$. coli, targeting and insertion into the membrane requires the signal recognition particle and the Sec pathway (Fekkes and Driessen 1999). In addition, YidC has been recognized to be re-

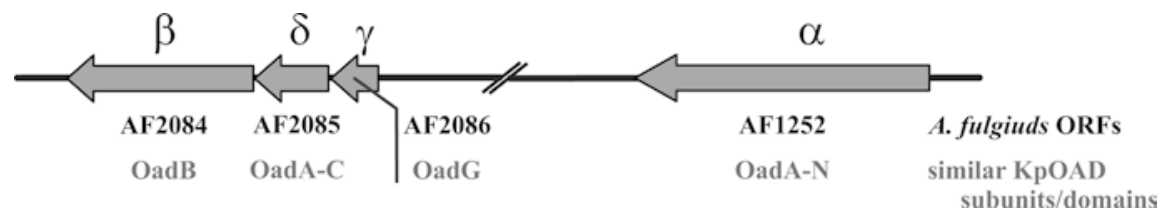


Table 2 Constructs for heterologous expression of oad genes of A. fulgidus in Escherichia coli

Construct

Yield (mg/g cells)

\begin{tabular}{|c|c|}
\hline Name & Comment \\
\hline pET16b-AfoadA + & $\begin{array}{l}\text { Encoding AfOadA includ } \\
\text { vector-encoded amino a }\end{array}$ \\
\hline pET16b-AfoadA & Encoding AfOadA includ \\
\hline pET24b-AfoadA & Encoding AfOadA includ \\
\hline pET43.1b-AfoadA & Encoding AfOadA includ \\
\hline pET24b-AfoadC & Encoding AfOadC incl \\
\hline pASK-AfoadB ${ }^{f}$ & $\begin{array}{l}\text { Encoding AfOadB includ } \\
\text { and a StrepTag }\end{array}$ \\
\hline pET16b-AfoadB & Encoding AfOadB includ \\
\hline pET24b-AfoadB & Encoding AfOadB includ \\
\hline pET24b-AfoadCB & Encoding AfOadCB \\
\hline pET24b-AfoadCBA & Encoding AfOadCBA \\
\hline pET24b-AfoadGCB & Encoding AfOadGCB \\
\hline pET24b-AfoadGCBA & Encoding AfOadGCBA \\
\hline
\end{tabular}

${ }^{\text {a }}$ Ten consecutive histidine residues followed by nine amino acids (SSGHIEGRH)

${ }^{\mathrm{b}}$ Most of the overproduced protein ( $\left.>99 \%\right)$ was present as inclusion bodies (approximately $100 \mathrm{mg} \mathrm{g}^{-1}$ cells)

${ }^{\text {c}} \mathrm{Six}$ consecutive histidine residues

${ }^{\mathrm{d}}$ Fusion with 491 amino acid NusA (NusTag) protein (Harrison 2000)

a)

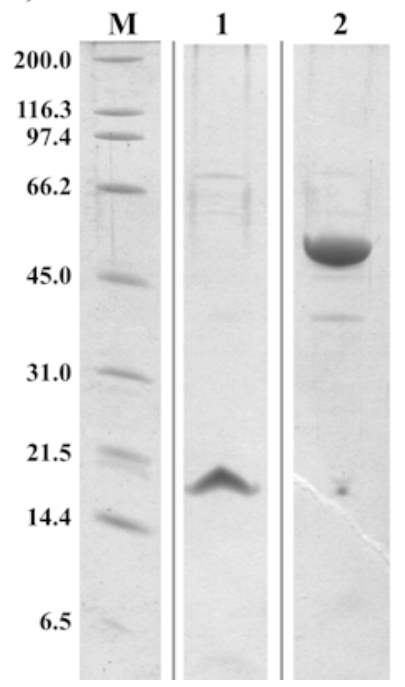

b)

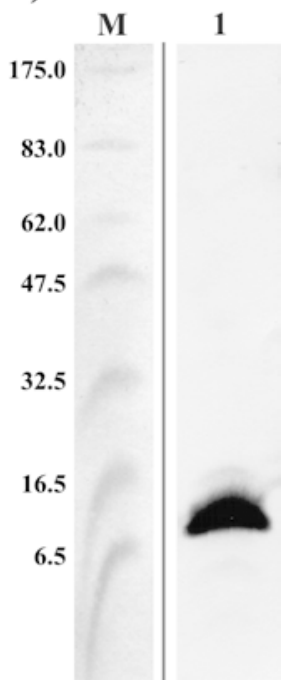

Fig. 2 SDS-PAGE of heterologously expressed AfOadC and AfOadA and Western blot of AfOadC. Each lane was loaded with $2 \mu \mathrm{g}$ of protein purified as described in "Materials and methods." a Silver-stained SDS-PAGE gels; $M$ BioRad Broad Range marker, lane 1 AfOadC purified via SoftLink-avidin column, lane 2 AfOadA purified via Ni-NTA-agarose column. b Western blot of AfOadC; $M$ NEB Prestained Broad Range marker, lane 1 AfOadC purified via Ni-NTA-agarose column. The blot was incubated with alkaline-phosphatase-conjugated avidin to show the biotinylation of AfOadC

quired for the insertion or proper folding of specific membrane proteins (Nagamori et al. 2004; Pohlschroder et al. 2004). If specific archeal targeting and folding proteins are required for the integration of archeal membrane proteins, this would prevent their successful synthesis in E. coli (Eichler 2000; Pohlschroder et al. 2004).

${ }^{\mathrm{e}}$ The values indicated are the yield of biotinylated protein (purified via SoftLink-avidin column). Twice the amount can be purified via $\mathrm{Ni}^{2+}$-NTA affinity chromatography (biotinylated and non-biotinylated species)

${ }^{\mathrm{f}}$ Derivative of pASK-IBA4 (Skerra and Schmidt 2000)

The A. fulgidus OadA subunit transfers $\mathrm{CO}_{2}$ from oxaloacetate to OadC

Transfer of the labeled carboxyl group from $\left[4-{ }^{14} \mathrm{C}\right]-$ oxaloacetate to the biotin group of AfOadC was measured in the purified AfOadA and AfOadC proteins. Figure 3 shows the kinetics of the carboxyl transfer with various combinations of carboxyltransferase and biotin carrier protein from $A$. fulgidus and $K$. pneumoniae. Incorporation of radioactivity into the biotin-containing $\mathrm{CO}_{2}$ acceptor domain was dependent on the carboxyltransferase and was found for each of the four pairs tested, albeit at different rates. With both $K$. pneumoniae proteins, the transfer was approximately two times faster than with the similar A. fulgidus pair. Surprisingly, the fastest rates were observed with AfOadA/KpOadA-C pair. ${ }^{14} \mathrm{CO}_{2}$ labeling of the KpOadA-N/AfOadC pair was slow and the steady state level was reduced to about one third compared to the other combinations of transferase and biotin carrier protein. Taken together, these experiments identify AfOadA as carboxyltransferase and AfOadC as the appropriate $\mathrm{CO}_{2}$ acceptor.

\section{Reconstitution of AfOadA from inclusion bodies}

To make at least part of the AfOadA deposited in inclusion bodies available for structural investigations, refolding into the native structure was attempted. For this purpose, the inclusion bodies were dissolved in $6 \mathrm{M}$ guanidinium chloride and then diluted into buffer containing appropriate additives to facilitate refolding (Lilie et al. 1998). Most of the solubilized protein precipitated during this procedure, but the residual protein was essentially pure AfOadA (Table 3 ). The recovery of 


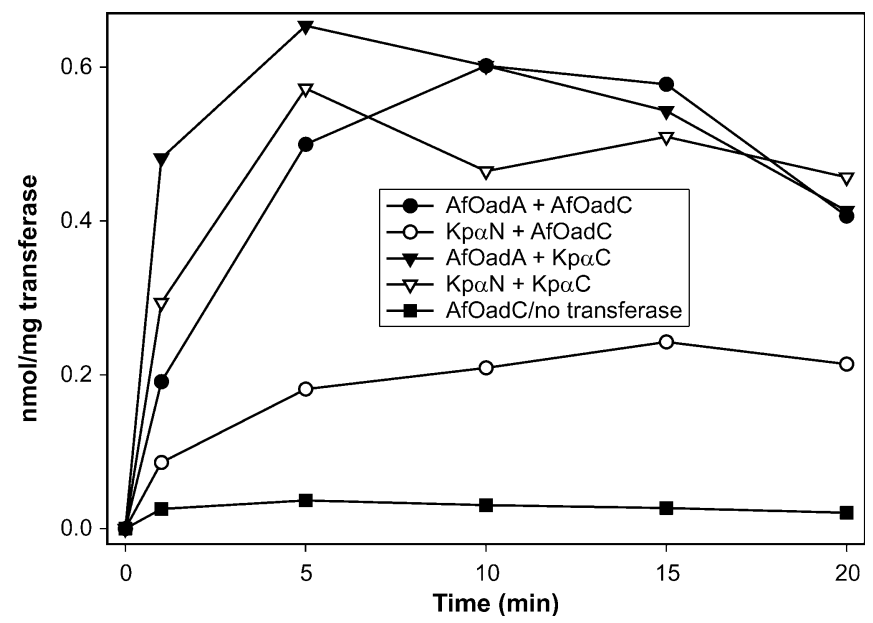

Fig. $3{ }^{14} \mathrm{CO}_{2}$ labeling of biotin carboxyl carrier proteins (BCC) by the carboxyl transferases from oxaloacetate decarboxylase of $K$. pneumoniae or A. fulgidus. The BCC subunits (AfOadC or KpOadA-C) were labeled as described in "Materials and methods." The experiments were carried out with the following combinations of BCC and transferase subunits: AfOadC and AfOadA (filled circles), AfOadC and KpOadA-N (open circles), KpOadA-C and AfOadA (filled inverted triangles), KpOadA-C and KpOadA-N (open inverted triangles). A control experiment was done with AfOadC only (filled squares)

Table 3 Refolding yields and recovered transferase activity of AfOadA. The recovered activity was calculated from the initial rates (nmol $\min ^{-1} \mathrm{mg}^{-1} \mathrm{OadA}$ ) as determined by the transferase activity assay. AfOadA inclusion bodies were prepared and solubilized with $6 \mathrm{M}$ guanidinium chloride. The yield was calculated from the amount of soluble protein present after dilution of the solubilized protein into refolding buffer. See "Materials and methods" for details

\begin{tabular}{lll}
\hline $\begin{array}{l}\text { AfOadA refolded in the } \\
\text { presence of }\end{array}$ & $\begin{array}{l}\text { Recovered specific } \\
\text { activity }(\%)\end{array}$ & $\begin{array}{l}\text { Yield } \\
(\%)\end{array}$ \\
\hline L-Arg & 25.4 & 11 \\
PPS & 30.3 & 8 \\
L-Arg $+0.1 \%$ LDAO & 37.6 & 18 \\
PPS + 0.1\% LDAO & 59.0 & 24 \\
$0.1 \%$ LDAO & 3.7 & 4 \\
No additive & 6.8 & 4 \\
Soluble AfOadA & 100 & - \\
\hline
\end{tabular}

transferase activity from inclusion bodies strongly depended on the additive. Without additives, only $4 \%$ of the inclusion bodies refolded into soluble protein. This refolded, soluble AfOadA showed only $7 \%$ of the specific transferase activity compared to soluble, cytoplasmically derived AfOadA. Using additives such as Larginine and PPS in the presence of $0.1 \%$ LDAO, yields could be dramatically improved (up to $24 \%$ ) and specific activity recovered to almost $60 \%$ compared to soluble expressed AfOadA (Table 3). These experiments impressively demonstrated the effectiveness of additives for refolding protein solubilized in guanidinium chloride and have provided us with the necessary amount of protein to initiate crystallization experiments.
Acknowledgments This work was supported by Swiss National Science Foundation. We thank Prof. Dr M. Thomm and Dr Harald Huber (Archaeenzentrum Universität Regensburg) for their generous gift of large amounts of $A$. fulgidus cells.

\section{References}

Ausubel FM, Brent R, Kingston RE, Moore DD, Seidman JG, Smith JA, Struhl K (1989) Short protocols in molecular biology, 1st edn. Greene Publishing Associates/Wiley-Interscience, New York

Balch WE, Fox GE, Magrum LJ, Woese CR, Wolfe RS (1979) Methanogens: reevaluation of a unique biological group. Microbiol Rev 43:260-296

Bott M (1997) Anaerobic citrate metabolism and its regulation in enterobacteria. Arch Microbiol 167:78-88

Bott M, Meyer M, Dimroth P (1995) Regulation of anaerobic citrate metabolism in Klebsiella pneumoniae. Mol Microbiol $18: 533-546$

Bott M, Pfister K, Burda P, Kalbermatter O, Woelke G, Dimroth P (1997) Methylmalonyl-CoA decarboxylase from Propionigenium modestum: cloning and sequencing of the structural genes and purification of the enzyme complex. Eur $\mathbf{J}$ Biochem 250:590-599

Buckel W (2001) Sodium ion-translocating decarboxylases. Biochim Biophys Acta 1505:15-27

Busch W, Saier MH Jr (2003) The IUBMB-endorsed transporter classification system. Methods Mol Biol 227:21-36

Di Berardino M, Dimroth P (1995) Synthesis of the oxaloacetate decarboxylase $\mathrm{Na}^{+}$pump and its individual subunits in Escherichia coli and analysis of their function. Eur $\mathbf{J}$ Biochem 231:790-801

Di Berardino M, Dimroth P (1996) Aspartate 203 of the oxaloacetate decarboxylase $\beta$ subunit catalyzes both the chemical and vectorial reaction of the $\mathrm{Na}^{+}$pump. EMBO J 15:1842-1849

Dimroth P (1986) Preparation, characterization, and reconstitution of oxaloacetate decarboxylase from Klebsiella aerogenes, a sodium pump. Methods Enzymol 125:530-540

Dimroth P (1997) Primary sodium ion translocating enzymes. Biochim Biophys Acta 1318:11-51

Eichler J (2000) Archaeal protein translocation crossing membranes in the third domain of life. Eur J Biochem 267:34023412

Fekkes P, Driessen AJ (1999) Protein targeting to the bacterial cytoplasmic membrane. Microbiol Mol Biol Rev 63:161-173

Hall TA (1999) BioEdit: a user-friendly biological sequence alignment editor and analysis program for Windows 95/98/NT. Nucleic Acids Symp Ser 41:95-98

Harrison RG (2000) Expression of soluble heterologous proteins via fusion with NusA protein. Innovations 11:4-7

Huder JB, Dimroth P (1993) Sequence of the sodium ion pump methylmalonyl-CoA decarboxylase from Veillonella parvula. J Biol Chem 268:24564-24571

Jockel P, Schmid M, Steuber J, Dimroth P (2000a) A molecular coupling mechanism for the oxaloacetate decarboxylase $\mathrm{Na}^{+}$ pump as inferred from mutational analysis. Biochemistry 39:2307-2315

Jockel P, Schmid M, Choinowski T, Dimroth P (2000b) Essential role of tyrosine 229 of the oxaloacetate decarboxylase $\beta$-subunit in the energy coupling mechanism of the $\mathrm{Na}^{+}$pump. Biochemistry 39:4320-4326

Klenk HP, Clayton RA, Tomb JF, White O, Nelson KE, Ketchum KA et al (1997) The complete genome sequence of the hyperthermophilic, sulphate-reducing archaeon Archaeoglobus fulgidus. Nature 390:364-370

Lilie H, Schwarz E, Rudolph R (1998) Advances in refolding of proteins produced in E. coli. Curr Opin Biotechnol 9:497-501

Miroux B, Walker JE (1996) Over-production of proteins in Escherichia coli: mutant hosts that allow synthesis of some 
membrane proteins and globular proteins at high levels. J Mol Biol 260:289-298

Nagamori S, Smirnova IN, Kaback HR (2004) Role of YidC in folding of polytopic membrane proteins. J Cell Biol 165:53-62

Pohlschroder M, Dilks K, Hand NJ, Rose RW (2004) Translocation of proteins across archaeal cytoplasmic membranes. FEMS Microbiol Rev 28:3-24

Sambrook J, Fritsch EF, Maniatis T (1989) Molecular cloning: a laboratory manual, 2nd edn. Cold Spring Harbor Laboratory, Cold Spring Harbor

Sanger F, Nicklen S, Coulson AR (1977) DNA sequencing with chain-terminating inhibitors. Proc Natl Acad Sci USA 74:54635467

Schmid M, Vorburger T, Pos KM, Dimroth P (2002a) Role of conserved residues within helices IV and VIII of the oxaloacetate decarboxylase $\beta$ subunit in the energy coupling mechanism of the $\mathrm{Na}^{+}$pump. Eur J Biochem 269:2997-3004
Schmid M, Wild MR, Dahinden P, Dimroth P (2002b) Subunit $\gamma$ of the oxaloacetate decarboxylase $\mathrm{Na}^{+}$pump: interaction with other subunits/domains of the complex and binding site for the $\mathrm{Zn}^{2+}$ metal ion. Biochemistry 41:1285-1292

Skerra A, Schmidt TG (2000) Use of the Strep-Tag and streptavidin for detection and purification of recombinant proteins. Methods Enzymol 326:271-304

Wendt KS, Schall I, Huber R, Buckel W, Jacob U (2003) Crystal structure of the carboxyltransferase subunit of the bacterial sodium ion pump glutaconyl-coenzyme A decarboxylase. EMBO J 22:3493-3502

Wild MR, Pos KM, Dimroth P (2003) Site-directed sulfhydryl labeling of the oxaloacetate decarboxylase $\mathrm{Na}^{+}$pump of Klebsiella pneumoniae: helix VIII comprises a portion of the sodium ion channel. Biochemistry 42:11615-11624 